\title{
A statistical framework to combine multivariate spatial data and physical models for hurricane surface wind prediction
}

\author{
Kristen M. Foley ${ }^{1}$, Montserrat Fuentes ${ }^{1 *}$ \\ ${ }^{1}$ Department of Statistics, North Carolina State University
}

\begin{abstract}
Storm surge is the onshore rush of seawater associated with hurricane force winds and can lead to loss of property and loss of life. Currently storm surge estimates are created by numerical ocean models that use a deterministic formula for surface wind fields. A new multivariate spatial statistical framework is developed to improve the estimation of these wind field inputs while accounting for potential bias in the observations. Using Bayesian inference methods we find that this spatial model improves estimation and prediction of surface winds and storm surge based on wind data for a case study of Hurricane Charley.
\end{abstract}

KeY Words: Bayesian inference; non-separable multivariate models; spatial statistics; storm surge forecasts; wind fields

*Correspondence to: M. Fuentes, Statistics Department, North Carolina State University (NCSU), Raleigh, NC 27695-8203, U.S.A.

E-mail: fuentes@stat.ncsu.edu

Contract/grant sponsor: National Science Foundation; contract/grant number: DMS 0353029. Contract/grant sponsor: National Oceanic and Atmospheric Administration through a Cooperative Agreement (Climate \& Weather Impacts on Society and the Environment) via the Charleston Coastal Services Center and the National Climate Data Center. 


\section{Introduction}

Estimating the spatial and temporal variation of surface wind stress fields plays an important role in modeling atmospheric and oceanic processes. This is particularly true for hurricane forecasting where numerical ocean models are used to simulate the coastal ocean response to the high winds and low pressure associated with hurricanes, such as the height of the storm surge and the degree of coastal flooding. According to the National Oceanic and Atmospheric Administration's (NOAA) Hurricane Research Division more than eighty-five percent of storm surge is caused by winds pushing the ocean surface ahead of the storm. The numerical ocean models used for storm surge forecasting are also driven primarily by the surface wind forcings. Houston and Powell (1994) did an analysis of the impact of wind field forcings on the storm surge forecasts used by the National Hurricane Center (NHC). They concluded that up to 6 hours before landfall, real-time model runs can be used to evaluate warnings and assess the extent of storm surge inundation. Houston et al. (1999) state that "an accurate diagnosis of storm surge flooding, based on the actual track and wind fields could be supplied to emergency management agencies, government officials, and utilities to help with damage assessment and recovery efforts." Our objective is to use all available sources of wind data in order to improve the estimation of the wind field inputs. It is expected that improving these

inputs will have a great impact on real-time storm surge estimates, allowing recovery efforts to be organized immediately after a hurricane has made landfall according to the areas most impacted by the storm.

Several studies have investigated the potential of using real-time observation-based winds to improve storm surge predictions as new wind observations have become available from the Hurricane Research Division (HRD). Since 1993 the HRD has reported real-time analyses of tropical cyclone surface wind observations for evaluation and eventually to be utilized by the NHC (Houston and Powell, 1994). Powell and Houston (1996) did a study of Hurricane Andrew (1996), and concluded that real-time wind fields would improve storm surge forecasts, particularly for storms with asymmetric (i.e. non axis-symmetric) wind fields. Houston et al. (1999) used the HRD winds as input into a two dimensional storm surge model. In their analysis they did not allow the wind and surface pressure field to change over time, but rather ran the ocean model assuming the wind 
and surface pressure fields were steady state for over 24 hours.

We propose a new method to use the HRD data to fit a statistical model for the winds that can be used as input into a numerical ocean model. The statistically-based winds are a function of the hurricane's location and can be updated at intervals as small as 10 minutes as more observations become available. A statistical framework is used to account for systematic and random errors in the observations while using these observations to model the surface winds as a multivariate spatial process. This is the first time that these methods have been applied to an ocean model application to be used for improved storm surge forecasting.

For coastal ocean modeling we use the Princeton Ocean Model (POM), which is adopted by NOAA as the operational coastal ocean forecasting system for the U. S. East Coast. POM is a fully three-dimensional numerical model and has the capability of modeling the evolution of ocean processes at many layers. In addition, POM includes open boundary conditions to account for surface wind stress and a bottom following sigma coordinate system to account for differences in water depth. As a result, the POM model is able to predict storm surge with greater spatial resolution and temporal accuracy than simpler models with very few layers or quasi-geostrophic dynamics which often cannot capture the large topographical variability of the coastal regions. Unlike global and other large scale forecasting models, this model can be applied at a higher resolution to a much smaller regional domain. Using statistical methods to combine observed data and model output at this smaller scale is very challenging but it will ultimately have the greatest impact on the health and safety of citizens living along the coast.

The statistical modeling of spatial processes in the atmospheric sciences has been an active area of research over the past decade. Hierarchical Bayesian methods have been found to be particularly useful for such applications, e.g. see Wikle et al. (1998), Berliner et al. (1999) and Royle et al. (1999). Such methods have been applied to modeling wind fields. Wikle et al. (2001) present a hierarchical spatio-temporal model for surface wind fields over the tropical oceans. Fuentes et al. (2005) propose a method for predicting coastal wind fields based on a non-stationary and non-separable covariance model that accounts for bias in available observations. In both of these studies the orthogonal components of the vector wind fields are treated as independent. Cripps et al. (2005) 
apply generalized autoregressive conditional heteroscedastic (GARCH) models to the bivariate wind vector at a single spatial location. In our statistical framework we combine all sources of available data including a physics-based wind model and the observational data currently available to NHC for coastal ocean prediction along the Eastern coastline. We investigate the importance of accounting for the cross-covariance of hurricane surface wind fields. The ultimate goal in the application presented here is to develop methods that would be practical (in terms of computing time) for a real-time forecast or nowcast scenario. Hence the sophistication of any proposed methodology must be constrained by the computing demands of the method. The scientific question of interest is how to balance the sophistication of the wind field model with computational cost.

Section 2 describes the deterministic physical model for surface wind fields, the available observed data and the proposed statistical model. Section 3 reviews statistical methodology for multivariate data following the linear model of coregionalization used in this paper. We outline a hierarchical Bayesian approach for estimation of parameters in a deterministic wind model and prediction of gridded surface wind fields. Section 4 describes the results of a case study for wind field data from Hurricane Charley in August of 2004. The estimated wind fields are used to initialize the numerical ocean model and are shown to improve storm surge prediction. A concluding discussion is given in section 5 .

\section{Data and Scientific Problem}

\subsection{Modeling Surface Wind Fields}

For coastal ocean modeling we use the Princeton Ocean Model (POM), which is a three-dimensional primitive equations ocean model (Blumberg and Mellor, 1987). Surface wind fields are the most important input variable used to initialize the numerical ocean model to simulate the coastal ocean response to hurricanes. A wind field is made up of wind vectors defined at a finite set of grid points such that each vector has a magnitude or wind speed value (typically measured in meters per second or knots) and a direction. These vectors are decomposed into orthogonal $u$ winds, (East-West) and, $v$ winds, (North-South). We use a Bayesian hierarchical framework to combine different sources 
of wind data at different locations in order to predict the wind fields at the grid locations used by the ocean model. Let $\mathbf{V}_{t}(\mathbf{s})=\left(u_{t}(\mathbf{s}), v_{t}(\mathbf{s})\right)^{T}$ be the underlying unobserved spatial process for the wind components at location $\mathbf{s}$ and time $t$. We use the standard Cartesian decomposition to define the wind vectors since the observed and predicted winds provided by NOAA are based on this decomposition. However we note that other decompositions are possible and in particular the $u$ and $v$ components could also be defined according to the direction of the motion of the storm at a given time.

Currently for storm surge forecasting a deterministic wind model is used, referred to as the Holland wind model (Holland, 1980) which determines the wind velocity induced by the hurricane pressure gradient under cyclostrophic balance. The Holland wind model for surface wind speed at a given location $\mathbf{s}$ is:

$$
W(\mathbf{s})=\left[\frac{B}{\rho}\left(\frac{R \max }{r}\right)^{B}\left(P_{n}-P_{c}\right) \exp ^{-(R \max / r)^{B}}\right]^{1 / 2}
$$

$P_{n}$ is the ambient pressure, $P_{c}$ is the hurricane central pressure, $\rho$ is the air density (fixed at $1.2 \mathrm{~kg}$ $m^{-3}$ ), and $r$ is the distance from the hurricane center to location $\mathbf{s}$.

Under the assumption that the hurricane wind field is axis-symmetric the $u$ and $v$ components can be determined from the wind speed:

$$
\begin{aligned}
& u^{H}(\mathbf{s})=W(\mathbf{s}) \sin \phi \\
& v^{H}(\mathbf{s})=W(\mathbf{s}) \cos \phi
\end{aligned}
$$

where $\phi$ is the inflow angle at site $\mathbf{s}$ across circular isobars toward the storm center. That is, for every location the covariates for $u$ and $v$ are the radius $(r)$, and angle $(\phi)$. The wind components are a nonlinear function of these covariates with parameters $\theta^{H}=(B, R \max )$. The forcing parameter $R \max$ is the radius of maximum sustained wind of the hurricane. In the Holland model the maximum possible wind speed in the storm is a function of the central pressure of the storm and the value $B$ which defines the shape of the pressure profile. Large values of B correspond to a wind field with very high wind speeds in a small region near the center of the storm. A smaller $B$ value 
will mean high wind speeds across a greater radius of the storm. These parameters are typically based on results from previous studies and are held constant for all forecasting time periods.

Although this physical model incorporates important information provided by the observed central pressure and the location of the eye of the storm there are known deficiencies in this formulation. For example, the Holland winds are symmetric around the storm center, however it is known that wind speeds are typically higher on the right hand side of the hurricane (with respect to the storm movement). Various adaptations of this physical model have been proposed. Xie et al. (2006) create an asymmetric model by incorporating data provided by the National Hurricane Center (NHC) guidance on the maximum radial extent of winds of a given threshold in the four quadrants of the storm. The NHC uses a parametric wind formula similar to the Holland model to force the ocean model. Another approach would be to use a coupled atmospheric-oceanic numerical model to simulate the surface winds at the boundary layer of the ocean model. Global mesoscale numerical weather forecasting models such as the Mesoscale Model (MM5) and the Weather Research and Forecasting Model (WRF) are capable of making forecasts for surface level wind fields. However these forecasts are not suitable for real-time forecasts of hurricane winds. Historically these models have been unable to accurately reproduce the intensity of hurricane force winds. More recent reports show that MM5 and WRF are able to obtain more realistic wind speeds when run at high resolution (on the order of $1 \mathrm{~km}$ grid size). However the CPU time required to produce these modeled winds prevents such model runs from being used in real-time applications. The best approach for regional storm surge forecasting is still using parametric hurricane models, as discussed in Xie et al. (2006). For further details on storm surge forecasting for the coastal ocean and estuarine systems see Xie et al. (2004), Peng et al. (2004), and references therein.

Here we focus on the operational methods and observational data currently available to the NHC for coastal ocean prediction along the Eastern coastline and incorporate this information into a statistical framework. In our analysis we provide a new tool to estimate the parameters of the Holland wind function. We include the Holland winds as the mean function of a spatial statistical model and incorporate a spatial covariance to account for any structures in observed hurricane surface winds not captured by the parametric wind fields. This approach to wind field modeling provides parameter estimates and standard errors as well as estimated wind fields through spatial 
prediction.

\subsection{Wind Data}

There are three sets of available wind data to be used in hurricane forecasting and hindcasting. Data provided by the National Hurricane Center (NHC) specifies the location of the storm center and the central pressure. The NHC provides forecasts every 6 hours on the location of the hurricane center (in degrees latitude/longitude) and sustained wind speeds (nautical miles) in different quadrants of the storm. For historical datasets such as these there is also "best" track information for the hurricane center and central pressure based on all available observations along the coast. The track information is necessary when creating wind fields based on the Holland wind model. We use linear interpolation to interpolate the best track information to estimate the storm center and central pressure at ten-minute time intervals.

In addition, data on wind speed and direction at over 20 buoy locations along the Eastern coast is available from NOAA's National Data Buoy Center (NDBC). For this study we use the ten minute average wind speed $(\mathrm{m} / \mathrm{s})$ adjusted to a common height of 10 meters above sea level and ten minute average wind direction. Ten minute averaging times are considered to be representative of the timescales typically associated with oceanic response to surface stress (Houston et al., 1999). The wind speed and direction are then converted to $u$ and $v$ components.

Finally, gridded wind field data is available from NOAA's Hurricane Research Division (HRD). These wind fields are a combination of surface weather observations from ships, buoys, coastal platforms, surface aviation reports, reconnaissance aircraft data, and geostationary satellites which have been processed to conform to a common framework for height $(10 \mathrm{~m})$, exposure (marine or open terrain over land) and averaging period (maximum sustained 1 minute wind speed). The processing and quality control of the data use accepted methods from micrometeorology and wind engineering and provide the best available near real-time gridded hurricane wind analyses. However it is important to note that validation studies have shown that the HRD wind analyses are highly variable in accuracy, depending on the quality and quantity of the observations used, and on the appropriateness of the underlying assumptions used to manipulate the observations. Houston et al. 
(1999) report the gridded wind fields have been found to have estimated errors of up to $10 \%-20 \%$. For this reason although buoy observations are used to create the HRD wind fields we use observed winds from the NDBC buoy network as a second data source to provide an estimate of the bias in the HRD analysis fields. For more information on the HRD data see Powell et al. (1996) and Powell and Houston (1996). HRD data is typically available at 3 hour intervals. HRD gridded fields are provided at a resolution of approximately $6 \mathrm{~km} \times 6 \mathrm{~km}$ with the number of grid points on the order of $10^{4}$.

\subsection{Statistical Model for Data}

We use a statistical framework to account for the difference in uncertainty associated with buoy and HRD analysis data. As described in the previous section the observed winds at the buoys are considered more reliable compared to the HRD analysis data which has been shown to have potentially large biases. This does not mean that no bias exists in the buoy data. For example wave distortion effects have been found to result in a negative bias when deriving wind stress from low-level anemonometers (Large et al., 1995). All of the buoy observations used in this study have anemometer heights that are at least five meters above mean sea level. In fact more than half of the sites are at heights greater than ten meters and so should not be influenced by surface waves. We cannot hope to accurately estimate the bias of both data sources but only the bias of the HRD data with respect to the buoy data. We take this approach since past studies suggest that the maginitude of the bias in the HRD data, which includes satellite and ship data, is larger than the potential bias in the buoys.

Let $\mathbf{V}_{t}^{a}(\mathbf{s})=\left(v_{t}^{a}(\mathbf{s}), u_{t}^{a}(\mathbf{s})\right)^{T}$ be the $u$ and $v$ components provided by the HRD analysis at time $t$ and location s. Let $\mathbf{V}_{t}^{b}(\mathbf{s})=\left(v_{t}^{b}(\mathbf{s}), u_{t}^{b}(\mathbf{s})\right)^{T}$ be wind data from the buoys time $t$ and location $\mathbf{s}$. For now we consider time fixed and suppress the time index. We model the observed data as a function of the underlying true (unobserved) wind process $\mathbf{V}(\mathbf{s})=(u(\mathbf{s}), v(\mathbf{s}))^{T}$. Then we model $\mathbf{V}(\mathbf{s})$ as a multivariate spatial process with a mean function equal to the nonlinear parametric Holland formula. This framework is depicted in Figure 1. The data model includes bias parameters, a(s) 


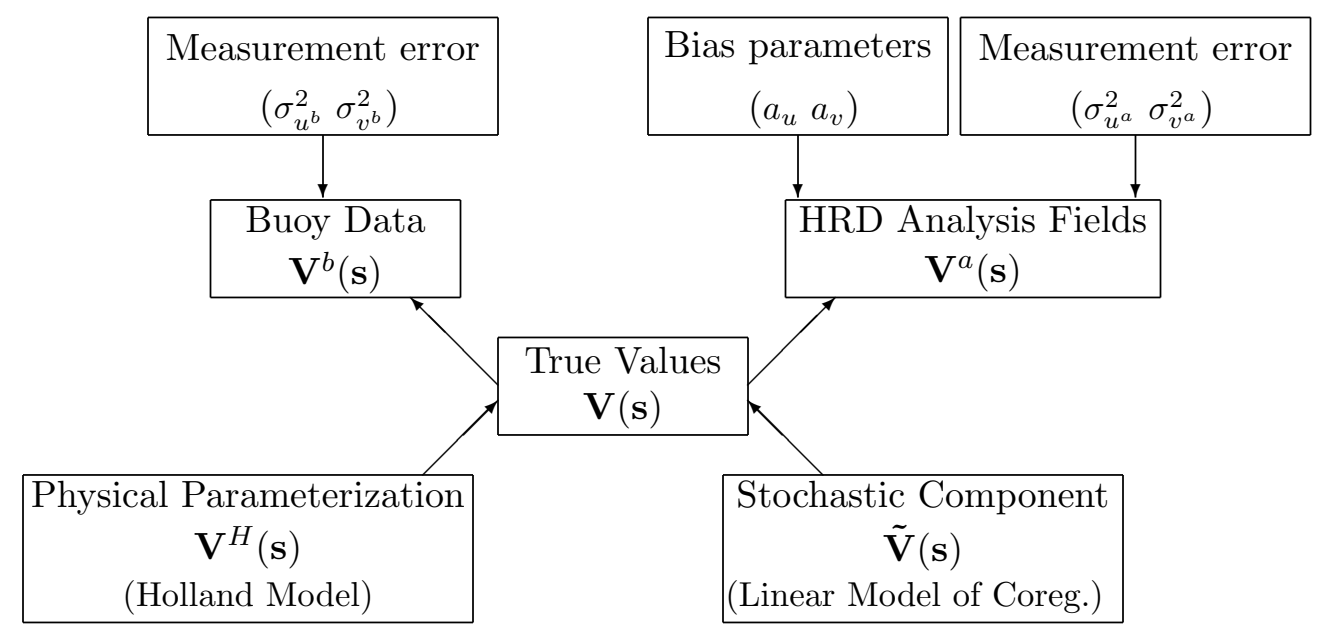

Figure 1: Model framework for wind components.

and $\mathbf{b}(\mathbf{s})$, as well as measurement error terms, $\epsilon^{b}(\mathbf{s})$ and $\epsilon^{a}(\mathbf{s})$ :

$$
\begin{aligned}
& \hat{\mathbf{V}}^{b}(\mathbf{s})=\mathbf{V}(\mathbf{s})+\epsilon^{b}(\mathbf{s}) \\
& \hat{\mathbf{V}}^{a}(\mathbf{s})=\mathbf{a}(\mathbf{s})+\mathbf{b}(\mathbf{s}) \mathbf{V}(\mathbf{s})+\epsilon^{a}(\mathbf{s})
\end{aligned}
$$

Here $a(\mathbf{s})=\left(a_{u}(\mathbf{s}), a_{v}(\mathbf{s})\right)^{T}$ and $b(\mathbf{s})=\operatorname{diag}\left(b_{u}(\mathbf{s}), b_{v}(\mathbf{s})\right)$ are spatial-temporal functions for additive and multiplicative bias. These may be modeled as polynomial functions of location, spline functions or as a function of additional covariates such as temperature or pressure. Based on exploratory analysis comparing the buoy and HRD data we find that a multiplicative bias term in not necessary and set $b_{u}(\mathbf{s}), b_{v}(\mathbf{s})=1$ for all locations $\mathbf{s}$. Due to the limited number of buoy data we consider only constant bias terms $a(\mathbf{s})=\left(a_{u}, a_{v}\right)^{T}$ for all locations $\mathbf{s}$.

We assume both measurement error processes, $\epsilon^{b}$ and $\epsilon^{a}$, are Gaussian white noise processes with $\epsilon^{b}(\mathbf{s}) \sim N\left(0, \Sigma^{b}\right)$, and $\epsilon^{a}(\mathbf{s}) \sim N\left(0, \Sigma^{a}\right)$ where $\Sigma^{b}=\operatorname{diag}\left(\sigma_{u^{b}}^{2}, \sigma_{v^{b}}^{2}\right)$ and $\Sigma^{a}=\operatorname{diag}\left(\sigma_{u^{a}}^{2}, \sigma_{v^{a}}^{2}\right)$. It is also assumed that these processes are independent of one another and the underlying process $\mathbf{V}(\mathbf{s})$. It is possible to instead model the measurement error as a spatially correlated process with some parametric spatial covariance function. We assume that the more complex spatial correlation structure is captured in the model for the underlying true process and hence there is no need for added complexity at this stage. 
The statistical model for the unobserved "true" wind fields combines physical information from the parametric Holland formula with a multivariate spatial process. The Holland equations (1) - (3) define axis-symmetric wind fields, $\mathbf{V}^{H}=\left(u^{H}, v^{H}\right)^{T}$, under cyclostrophic balance and represent the large scale or mean structure of the surface winds. A multivariate spatial process, $\tilde{\mathbf{V}}=(\tilde{u}, \tilde{v})^{T}$, is used to model the variability of the observed data that cannot be captured by any parameterization of the Holland formula. We define the overall model for the underlying wind vector as:

$$
\mathbf{V}(\mathbf{s})=\mathbf{V}^{H}(\mathbf{s})+\tilde{\mathbf{V}}(\mathbf{s})
$$

We can obtain more reliable wind fields either by (1) introducing a more sophisticated deterministic wind model for $\mathbf{V}^{H}$ (e.g. Xie et al. (2006)) (2) developing a sophisticated statistical multivariate spatial model for the stochastic component $\tilde{\mathbf{V}}$. The proposed approach needs to be run in real-time so a balance between (1) and (2) is the ultimate solution to the forecasting problem. Alternatively, one could write a stochastic version of the deterministic model and approximate the physical model using a stochastic spatial basis. This is the approach of Wikle et al. (2001) for oceanic surface winds. Since the operational storm surge model uses parametric wind fields (essentially like the Holland winds), it is preferable to instead provide tools to better estimate the parameters of the Holland model accounting for uncertainty in the parameter estimates. This motivates the problem of defining a multivariate spatial model for the stochastic component. We adopt this approach here and evaluate the improvement in the wind prediction and storm surge forecast.

\section{Modeling Multivariate Data}

\subsection{Linear Model of Coregionalization (LMC)}

In our setting, we account for any cross-dependency in the $u$ and $v$ winds by using a multivariate spatial model based on a linear model of coregionalization. This approach was first introduced as a method of dimension reduction in order to model a set of $p$ multivariate observations as a function 
of $k$ unobservable factors with $k<p$. More recently, Schmidt and Gelfand (2003), Banerjee et al. (2004), and Gelfand et al. (2004) proposed using this approach to construct valid cross-covariance functions by modeling a set of dependent spatial processes as a linear combination of independent spatial processes. We briefly describe the LMC model followed by a description of the steps for Bayesian inference and prediction based on the available multivariate wind data.

We assume we are working with multivariate Gaussian processes. We find this is a reasonable assumption based on analysis of the observed $u$ and $v$ velocities after subtracting off the Holland mean function. In general, consider modeling $p$ spatial processes $\tilde{\mathbf{V}}(\mathbf{s})_{(p \times 1)}$ for $\mathbf{s} \in \mathcal{D} \subset \mathcal{R}^{2}$. For a finite set of locations $\mathbf{s}_{1}, \ldots, \mathbf{s}_{n}$, let $\tilde{\mathbf{V}}$ denote a $n p \times 1$ vector of the the $p$ spatial functions evaluated at these $n$ sites: $\left\{\tilde{\mathbf{V}}: \tilde{V}_{1}\left(\mathbf{s}_{1}\right), \ldots, \tilde{V}_{1}\left(\mathbf{s}_{n}\right), \ldots, \tilde{V}_{p}\left(\mathbf{s}_{1}\right), \ldots, \tilde{V}_{p}\left(\mathbf{s}_{n}\right)\right\} . \tilde{V}_{j}\left(\mathbf{s}_{i}\right)$ denotes the $j^{\text {th }}$ spatial process of interest at location $\mathbf{s}$ with $j=1, \ldots, p$ and $i=1, \ldots, n$.

Let $w_{j}(\mathbf{s})$ be $p$ latent independent Gaussian spatial processes with mean zero. Each process has covariance function $\operatorname{Cov}\left(w_{j}\left(\mathbf{s}_{i}\right), w_{j}\left(\mathbf{s}_{i^{\prime}}\right)\right)=\rho_{j}\left(\mathbf{s}_{i}, \mathbf{s}_{i^{\prime}} ; \phi_{j}\right)$ with parameters $\phi_{j}, j=1, \ldots, p$. These covariance functions need not be stationary or isotropic. Now for $\mathbf{w}(\mathbf{s})=\left(w_{1}(\mathbf{s}), \ldots, w_{p}(\mathbf{s})\right)^{T}$ the stochastic component in (6) is modeled as:

$$
\tilde{\mathbf{V}}(\mathbf{s})=\mathbf{A w}(\mathbf{s})
$$

where $\mathbf{A}$ is a $p \times p$ weight matrix that determines the covariance between the $p$ variables. Without loss of generality we assume $\mathbf{A}$ is a full rank lower triangular matrix. This implies $E(\tilde{\mathbf{V}}(\mathbf{s}))=\mathbf{0}$ and

$$
\operatorname{Cov}\left(\tilde{\mathbf{V}}\left(\mathbf{s}_{i}\right), \tilde{\mathbf{V}}\left(\mathbf{s}_{i^{\prime}}\right)\right)=\sum_{j=1}^{p} \rho_{j}\left(\mathbf{s}_{i}, \mathbf{s}_{i^{\prime}} ; \phi_{j}\right) \mathbf{T}_{j}
$$

where $\mathbf{T}_{j}=\mathbf{a}_{j} \mathbf{a}_{j}^{T}$ and $\mathbf{a}_{j}$ is the $j^{\text {th }}$ column of $\mathbf{A}$. Thus the cross-covariance is non-stationary, depending on the form of the $\rho_{j}$. Also the cross-covariance function is non-separable, meaning we cannot separate the between site variability and the variability between measures taken at the same site.

$$
\operatorname{Cov}(\tilde{\mathbf{V}}, \tilde{\mathbf{V}})=\sum_{j=1}^{p} \mathbf{T}_{j} \otimes \mathbf{R}_{j}
$$

for $\mathbf{R}_{j}$ a set of $p n \times n$ matrices with elements $\left(R_{j}\right)_{i i^{\prime}}=\rho_{j}\left(\mathbf{s}_{i}, \mathbf{s}_{i^{\prime}} ; \phi_{j}\right)$ and $\otimes$ denotes the Kronecker 
product. (Note, for $\tilde{\mathbf{V}}=\left(\tilde{V}\left(\mathbf{s}_{1}\right), \tilde{V}\left(\mathbf{s}_{2}\right), \ldots, \tilde{V}\left(\mathbf{s}_{n}\right)\right)^{T}$ then the covariance is simply, $\operatorname{Cov}(\tilde{\mathbf{V}}, \tilde{\mathbf{V}})=$ $\left.\sum_{j=1}^{p} \mathbf{R}_{j} \otimes \mathbf{T}_{j}.\right)$ For $\rho_{j}$ stationary correlation functions, $\sum_{j=1}^{p} \mathbf{T}_{j}=\mathbf{T}$ is the covariance matrix for $\tilde{\mathbf{V}}(\mathbf{s})$ so there is a one-to-one relation between matrix $\mathbf{A}$ and matrix $\mathbf{T}$. For $p=2$,

$$
\mathbf{T}=\left(\begin{array}{cc}
A_{11}^{2} & A_{11} A_{21} \\
A_{11} A_{21} & A_{21}^{2}+A_{22}^{2}
\end{array}\right)
$$

Gelfand et al. (2004) also propose a non-stationary version of the LMC model by allowing the weight matrix to be a spatial function, $\mathbf{A}(\mathbf{s})$. Since there is a one-to-one correspondence between $\mathbf{A}(\mathbf{s})$ and the covariance matrix $\mathbf{T}(\mathbf{s})$, they develop an Spatial Wishart distribution for

$\mathbf{T}(\mathbf{s})$. However this leads to a nonstandard distribution for $\tilde{\mathbf{V}}$, e.g. the expression in (9) for the cross-covariance is no longer valid since the weight matrix is now a random spatial process. We thus propose to model all of the spatial structure within the latent processes. This will yield a non-stationary model for $\mathbf{V}(\mathbf{s})$ whose properties are easier to derive and interpret.

Note that a separable covariance model is just a special case of the LMC model where $\rho_{j}=\rho_{j^{\prime}}$ for all $j, j^{\prime}=1, \ldots, p$ and $\rho_{j}$ a stationary correlation function. In this case, $\operatorname{Cov}(\tilde{\mathbf{V}}, \tilde{\mathbf{V}})=\mathbf{T} \otimes \mathbf{R}$ for $(R)_{i i^{\prime}}=\rho\left(\mathbf{s}_{i}, \mathbf{s}_{i^{\prime}} ; \phi\right)$. This is referred to as an intrinsic multivariate correlation model because the correlation structure of the set of variables is independent of the spatial correlation. That is, the correlation between two variables does not depend on spatial scale (Wackernagel, 1998):

$$
\operatorname{Corr}\left(\tilde{V}_{j}\left(\mathbf{s}_{i}\right), \tilde{V}_{j^{\prime}}\left(\mathbf{s}_{i^{\prime}}\right)\right)=\frac{T_{j j^{\prime}} \rho\left(\mathbf{s}_{i}, \mathbf{s}_{i^{\prime}} ; \phi\right)}{\sqrt{T_{j j} \rho\left(\mathbf{s}_{i}, \mathbf{s}_{i^{\prime}} ; \phi\right) T_{j^{\prime} j^{\prime}} \rho\left(\mathbf{s}_{i}, \mathbf{s}_{i^{\prime}} ; \phi\right)}}=\frac{T_{j j^{\prime}}}{\sqrt{T_{j j} T_{j^{\prime} j^{\prime}}}}
$$

In the following application we apply both a separable and non-separable LMC model to the surface wind fields.

\subsection{Statistical Methodology}

We now turn to the issue of estimation and prediction of the wind fields to be used in the ocean model application. In order to estimate the wind field at a given set of locations we need estimates 
for the parameters $\boldsymbol{\Theta}=\left(\theta^{D}, \theta^{H}, \tilde{\theta}\right)$ where $\theta^{D}=\left(a_{u}, a_{v}, \sigma_{b}^{2}, \sigma_{a}^{2}\right)$ are the bias and measurement error parameters, $\theta^{H}=(B, R \max )$ are the parameters of the Holland model and $\tilde{\theta}=\left(A,\left\{\phi_{j}\right\}\right)$ are the parameters for the LMC model for $j=1,2$. We present a Bayesian framework that allows for estimation of the parameters of the multivariate spatial model and the physically based parameterized wind function while accounting for potential additive bias in the observed data. Let $\hat{\mathbf{V}}_{t}=\left(\hat{\mathbf{V}}_{t}^{b}, \hat{\mathbf{V}}_{t}^{a}\right)$ be all the available wind data at time $t$ from the buoys and HRD analysis wind fields. The posterior predictive distribution for the wind vector $\left(u\left(\mathbf{s}_{0}\right), v\left(\mathbf{s}_{0}\right)\right)^{T}$ at a particular location $\mathbf{s}_{0}$ at time $t$ is:

$$
P\left(\mathbf{V}\left(\mathbf{s}_{0}\right) \mid \hat{\mathbf{V}}\right) \propto \int P\left(\mathbf{V}\left(\mathbf{s}_{0}\right) \mid \hat{\mathbf{V}}, \boldsymbol{\Theta}\right) \times P(\boldsymbol{\Theta} \mid \hat{\mathbf{V}}) d \mathbf{\Theta}
$$

The full posterior predictive distribution (12) conditioned on the data from the $n_{a}$ locations of the analysis wind fields and the $n_{b}$ buoy sites can be approximated by using Markov Chain Monte Carlo. We use a Blocking Gibbs Sampling algorithm (within the software WinBUGS, Spiegelhalter et al. (1996)) to sample from the posterior distribution of the parameters by alternating between the parameters of the underlying process, $\left(\theta^{H}, \tilde{\theta}\right)$, and the parameters for the measurement error and bias, $\theta^{D}$. Our Gibbs algorithm proceeds in the following stages.

\section{Stage 1:}

Conditioned on the true wind process $\mathbf{V}$ we obtain the posterior distribution of the parameters, $\theta^{D}$, that explain the bias and uncertainty about the data. The posterior distribution will be completely specified once we define the priors for $\theta^{D}$ since we have:

$P\left(\hat{\mathbf{V}}^{b} \mid \mathbf{V}, \sigma_{b}^{2}\right) \sim N\left(\mathbf{V}, \Sigma^{b}\right)$

$P\left(\hat{\mathbf{V}}^{a} \mid \mathbf{V}, a_{u}, a_{v}, \sigma_{a}^{2}\right) \sim N\left(\left(a_{u}, a_{v}\right)^{T}+\mathbf{V}, \Sigma^{a}\right)$

\section{Stage 2:}

At the second stage we use the statistical model for the underlying true winds. Let $\mathbf{V}^{H}$ denote the output from the Holland model with parameters $\theta^{H}$ at the locations of the observed data. Thus based on the conditional distribution:

$P\left(\mathbf{V} \mid \theta^{H}, \tilde{\theta}, \mathbf{V}^{H}\right) \sim N\left(\mathbf{V}^{H}, \sum_{j=1}^{2} \mathbf{T}_{j} \otimes \mathbf{R}_{j}\right)$

and priors for $\left(\theta^{H}, \tilde{\theta}\right)$, we obtain the posterior distribution for the parameters in the Holland model and for the parameters in the stochastic model. 


\section{Stage 3:}

We simulate values of $\mathbf{V}$, the unobserved true process, at the $n_{a}$ locations of the analysis wind fields and the $n_{b}$ buoy sites conditioned on values of $\boldsymbol{\Theta}$ updated in the previous two stages. Let $N=n_{b}+n_{a}$. The conditional multivariate distribution of $\mathbf{V}$ at a new location $\mathbf{s}_{0}$ is:

$$
P\left(\mathbf{V}\left(\mathbf{s}_{o}\right) \mid \hat{\mathbf{V}}, \boldsymbol{\Theta}\right) \sim N\left(\mathbf{V}^{H}\left(\mathbf{s}_{o}\right)+\tau^{T} \Sigma^{-1}[\hat{\mathbf{V}}-\hat{\mu}], \hat{T}-\tau^{T} \Sigma^{-1} \tau\right)
$$

where $\tau=\hat{\operatorname{Cov}}\left(\mathbf{V}\left(\mathbf{s}_{o}\right), \hat{\mathbf{V}}\right)$ is a $(2 N \times 2)$ matrix and $\Sigma_{(2 N \times 2 N)}=\hat{\operatorname{Cov}}(\hat{\mathbf{V}}, \hat{\mathbf{V}}) \cdot \hat{\mu}=\left(\mathbf{V}^{H}, \mathbf{a}+\mathbf{V}^{H}\right)^{T}$ is the Holland mean function evaluated at the buoy and HRD locations accounting for the bias in the HRD dataset. For more details on combining different sources of spatial data for Bayesian prediction see Fuentes and Raftery (2005).

Thus after iterating through the three stages we have $M$ samples from the posterior distributions

for the parameters, $\left\{\boldsymbol{\Theta}^{(m)}\right\}, m=1, \ldots, M$. The Rao-Blackwellized estimator (Gelfand and Smith, 1990) for (12) is

$$
P\left(\mathbf{V}\left(\mathbf{s}_{o}\right) \mid \hat{\mathbf{V}}\right)=\frac{1}{M} \sum_{m=1}^{M} P\left(\mathbf{V}\left(\mathbf{s}_{o}\right) \mid \hat{\mathbf{V}}, \Theta^{(m)}\right)
$$

\section{Prior Specification}

Uniform priors are used for the random effects such that $\sigma_{b} \sim \operatorname{Uniform}(.01,1)$ and $\sigma_{a} \sim \operatorname{Uniform}(.01,5)$. We use relatively informative priors for the buoy data here based on previous experience and analysis of similar datasets. These hyperprior values are based on information reported by the NDBC on the accuracy of the buoy equipment. The bias parameters are assigned normal priors with $a_{u}, a_{v} \sim N(0, .001)(.001$ is the precision). Priors for the process parameters, $\theta^{H}=(B, R \max )$, are chosen based on previous studies. $B \sim$ Uniform $(1,2.5)$, $R \max \sim$ Uniform $(15,55)$ based on the physical constraints described earlier and discussed in Holland (1980). In the next section we describe the spatial covariance used for the hurricane case study. For our application, $\phi_{1}, \phi_{2}$ are range parameters which we assign noninformative uniform priors, Uniform $(10,1000)$, based on the size of the domain (in $\mathrm{km}$ ) for the data.

Finally we must specify a prior for the weight matrix A. We take advantage of a conditional or hierarchical modeling approach described in Schmidt and Gelfand (2003) in order to ease the 
computational cost of working with the full $n p \times n p$ cross-covariance matrix of the stochastic component, $\operatorname{Cov}(\tilde{\mathbf{V}}, \tilde{\mathbf{V}})=\sum_{j=1}^{p} \mathbf{T}_{j} \otimes \mathbf{R}_{j}$, in Stage 2 of the algorithm. Evaluating (9) for $p=2$, the joint covariance for the underlying true process $\mathbf{V}$ can be written as:

$$
\operatorname{Cov}(\mathbf{V}, \mathbf{V})=\left(\begin{array}{cc}
A_{11}^{2} \mathbf{R}_{1} & A_{11} A_{21} \mathbf{R}_{1} \\
A_{11} A_{21} \mathbf{R}_{1} & A_{21}^{2} \mathbf{R}_{1}+A_{22}^{2} \mathbf{R}_{2}
\end{array}\right)
$$

Now following a standard multivariate normal result:

$$
\begin{aligned}
\mathbf{u} & \sim N\left(\mathbf{u}^{H}, A_{11}^{2} \mathbf{R}_{\mathbf{1}}\right) \\
\mathbf{v} \mid \mathbf{u} & \sim N\left(\mathbf{v}^{H}+\frac{A_{21}}{A_{11}}\left(\mathbf{u}-\mathbf{u}^{H}\right), A_{22}^{2} \mathbf{R}_{2}\right)
\end{aligned}
$$

Using (10) we can rewrite (16)-(17) in terms of the elements of the $\mathbf{T}$ matrix. Under the new conditional parameterization we assign the scale parameters $A_{11}^{2}=T_{11}$ and $A_{22}^{2}=T_{22}-\frac{T_{12}^{2}}{T_{11}}$

inverse gamma distributions $\left(\frac{1}{A_{11}^{2}}, \frac{1}{A_{22}^{2}} \sim \operatorname{Gamma}(.01, .01)\right)$ and the weight parameter $\frac{A_{21}}{A_{11}}=\frac{T_{12}}{T_{11}} \sim$ $N(0, .001)$ (.001 is the precision). A similar approach can be used for multivariate data with $p>2$. See the Schmidt and Gelfand (2003) study of multivariate air pollutant data for the conditional and unconditional models for $p=3$.

\section{Analysis of Data from Hurricane Charley}

In this section we apply the multivariate statistical model for hurricane surface winds to a case study of Hurricane Charley of 2004. This case study was chosen because the hurricane surface winds induced measurable storm surge along the coast of Georgia and the Carolinas. We compare four different statistical models through a series of model diagnostics and look at posterior parameter estimates for the separable LMC model for 6 time periods. An empirical Bayesian estimation procedure is used for creating the gridded wind field inputs for the ocean model. Finally we run the numerical ocean model using the estimated wind fields and compare the results to the predicted surge based on the Holland winds. 
Hurricane Charley crossed over the central Florida peninsula and moved offshore early on the morning August 14th, 2004. Charley made landfall at Cape Romain, SC at 1400 UTC (Coordinated Universal Time) on the 14th as a weakening Category 1 storm with highest winds around 80 miles per hour $(70 \mathrm{kt} / \mathrm{hr})$. It then moved off shore again only to make landfall a few hours later at North Myrtle Beach, SC. Water level gauges along the coast of Georgia and the Carolinas reported storm surge heights up to 1.5 meters above normal tidal levels. We use gridded wind field data from the Hurricane Research Division for 6 times on August 14th (0200, 430, 730, 1030, 1330, and 1630 UTC) leading up to Hurricane Charley's landfall in South Carolina. For computational reasons we sub-sample the gridded HRD data to reduce the number of grid points to the order of $10^{2}$. Buoy observations from 23 locations are also used at these time periods to estimate potential bias in the HRD data.

\subsection{Model Estimation for HRD and Buoy Data}

Our objective is to fit a joint model for the wind field vectors and obtain the posterior predictive distribution of the winds at the ocean model grid points. Using the software GeoBugs (Spiegelhalter et al., 1996), we fit four different models using 225 locations of the HRD gridded data for $u$ and $v$ and data from the 23 buoys. Model 1 uses only the Holland mean function, i.e. $\tilde{\mathbf{V}}(\mathbf{s})$ is neglected. Note that in this case the parameters of the Holland winds are estimated using our framework rather than fixed values based on "expert knowledge". Model 2 treats the $u$ and $v$ components as independent so that $T_{12}=A_{11} A_{21}=0$ in equation 15. Model 3 is a separable LMC model outlined in section 3.1 so that the parameters of the underlying spatial processes are assumed to be equal (i.e. $\phi_{1}=\phi_{2}$ ). Finally, Model 4 is a non-separable LMC model which does not impose this assumption about the spatial processes.

For this application, we assume $\rho_{j}\left(\mathbf{s}_{i}-\mathbf{s}_{i^{\prime}} ; \phi_{j}\right)$ are stationary correlation functions with parameters $\phi_{j} ; j=1,2$. We did allow for geometric anisotropy, e.g. Fuentes et al. (2005), but found no significant evidence of a lack of isotropy in the $u$ and $v$ winds for this case. Here we use an expo-

nential correlation function such that $\rho_{j}\left(h ; \phi_{j}\right)=\exp \left(-h / \phi_{j}\right), j=1,2$ for $h=\left\|\mathbf{s}_{i}-\mathbf{s}_{i^{\prime}}\right\|$ and the range parameter $\phi_{j}$ represents the distance at which two wind measurements become practically 
uncorrelated.

Table 1: Posterior medians and 2.5 and 97.5 percentiles for the Holland mean function in the separable LMC spatial model for $u$ and $v$.

\begin{tabular}{rrrrrrr}
\hline \multicolumn{3}{c}{ B } & \multicolumn{3}{c}{ Rmax } \\
\hline UTC & $2.5 \%$ & Median & $97.5 \%$ & $2.5 \%$ & Median & $97.5 \%$ \\
\hline 200 & 1.62 & 2.29 & 2.50 & 5.00 & 5.10 & 5.90 \\
430 & 1.04 & 1.35 & 1.83 & 5.86 & 7.11 & 8.69 \\
730 & 1.27 & 1.65 & 2.22 & 20.65 & 23.39 & 28.43 \\
1030 & 1.00 & 1.35 & 2.45 & 6.65 & 12.96 & 27.44 \\
1330 & 1.00 & 1.13 & 1.60 & 7.46 & 10.84 & 27.45 \\
1630 & 1.46 & 1.91 & 2.45 & 5.41 & 7.25 & 10.87 \\
\hline
\end{tabular}

Table 1 shows the posterior distribution summaries of the Holland parameters in the separable LMC model for $u$ and $v$. The parameter estimates are significantly different for different time periods. Standard values for the Holland model are $B=1.9$ and $R \max =35$ to $55 \mathrm{~km}$ depending on the size and intensity of the storm, e.g. see Hsu and Yan (1998). This range of values for Rmax based on previous studies does not capture any of the values estimated in this case study. At 200 and 430 UTC the hurricane has just passed over Florida and is less symmetric. The radius of maximum winds $(R \max )$ at these times is 5 to $7 \mathrm{~km}$. Using the standard values greatly overestimates the strength and extent of the winds at such times when the storm is less organized. We also note that the cross-correlation values $\left(T_{12} / \sqrt{T_{11} T_{22}}\right)$ range from .24 to -.23 across the six time periods. The posterior intervals for the correlation do not contain zero, suggesting there is significant correlation between the $u$ and $v$ winds at all time periods.

\section{Model Diagnostics and Calibration}

The Deviance Information Criterion (DIC) (Spiegelhalter et al., 2002) shown in Table 2 is used to evaluate the different statistical models (where lower DIC values are better). All of the spatial models perform much better than the model with just the Holland mean function. The multivariate LMC models fit the data better for all of the times except 430 and 1630 UTC. At 430 UTC the DIC 
values for the independent and nonseparable models are similar. At 1630 UTC the independent model appears to be the better fit. At 430 and 1630 UTC the eye of the hurricane is directly off the coast and the wind speed contours appear more symmetric than the other time periods. Thus although it is clear that the spatial models do a much better job of estimating the wind fields it appears that the issue of independence of the $u$ and $v$ velocity components depends on the structure and organization of the storm at a given time.

Table 2: Deviance Information Criterion (DIC) Values

\begin{tabular}{rrrrrrr}
\hline Model & 200 UTC & 430 UTC & 730 UTC & 1030 UTC & 1330 UTC & 1630 UTC \\
\hline Model 1 & 3151.8 & 3400.9 & 3433.7 & 3541.7 & 3147.8 & 3353.7 \\
Model 2 & 2827.6 & 1402.9 & 1419.4 & 1551.7 & 1522.5 & 1939.2 \\
Model 3 & 2093.6 & 1505.9 & 1292.4 & 1023.7 & 1381.2 & 2167.1 \\
Model 4 & 1832.5 & 1417.3 & 1093.9 & 1285.0 & 1439.4 & 2547.1 \\
\hline
\end{tabular}

We use cross validation to evaluate how well the models predict winds at a new location. Models 1 through 4 are used to predict at thirty HRD grid points that were not used for parameter estimation. The median values of the posterior predictive distributions are used to compare to the observed values at the hold out sites. Analysis of the cross-validation errors under each model shows no significant prediction bias for any of the models. The non-spatial model using only the Holland mean function has much larger error variance and several outlier values show that the winds at a few locations are underpredicted by up to 15 to $20 \mathrm{~m} / \mathrm{s}$. The root means square prediction error (RMSPE) values for Models 2, 3 and 4 (the spatial models) are very similar. We compare the calibration for each model and each time period based on the $95 \%$ posterior predictive intervals. For the models to be well calibrated the observed velocities should fall within the posterior predictive intervals at least $95 \%$ of the time. Figure 2 shows calibration plots for the $v$ component for the hold out sites at 430UTC. The prediction results for Models 2, 3 and 4 are practically identical. Similar results were found for the other time periods and for the $u$ winds. All of the spatial models have calibration percents greater than or equal to $95 \%$ at all times. However the calibration percents for Model 1 (the non-spatial model) are lower, ranging from $86.7 \%$ to $96.7 \%$. Thus although the DIC values show a clear difference in estimation results across the four models, it appears that Models 
2, 3 and 4 perform well in terms of prediction. In fact it is not surprising that although the three spatial models result in different covariance parameter estimates this does not have a dramatic impact on spatial prediction (Stein, 1999).

\subsection{Ocean Model Application}

We now consider the application of the proposed wind field model for storm surge prediction. For coastal ocean modeling we use the Princeton Ocean Model (POM), which is a three-dimensional primitive equations ocean model that uses a finite differencing technique to solve the governing equations and boundary equations. It has been designed to represent ocean phenomena of 1-100km length and tidal-monthly time scales (Blumberg and Mellor, 1987). POM has been used in various applications to model estuaries and bayes, semienclosed seas and coastal regions around the world and has been applied extensively for studies in the Gulf Stream region (see Ezer and Mellor (1997) and references therein).

In the previous section we used the fully Bayesian solution for the posterior predictive distribution $P\left(\mathbf{V}\left(\mathbf{s}_{o}\right) \mid \hat{\mathbf{V}}, \Theta\right)$ outlined in section 3.2 to predict at 30 cross validation sites for six different time periods. However, for the storm surge application we must create predicted wind fields for every grid point of the ocean model domain for 96 ten-minute time increments over a period from 200 UTC to 1750 UTC on August 14, 2004. For this application the ocean model is typically run at a resolution of 2 to $4 \mathrm{~km}$ grids. In our case the model was run using a resolution of 2 minute longitude by 2 minute latitude grid $(\approx 3.1 \mathrm{~km}$ by $3.7 \mathrm{~km})$ with a total of $151 \times 115$ grid points. Hence it is computationally infeasible to use the fully Bayesian prediction to predict the winds at a such a high resolution.

Here we take an empirical Bayesian approach and use the posterior median values of the parameters, $\boldsymbol{\Theta}$, as the fixed "true" parameter values. Then the predictions are made following the conditional distribution in equation (13). We compare the two methods based on the 30 cross validation sites used in the previous section. Results show that the coverage is almost identical for both the fully Bayes and empirical Bayes prediction. Also, on average, the widths of the fully Bayesian credible intervals are only $4 \%$ larger than the intervals based on the empirical approach. 
Figure 2: Cross Validation 430 UTC: Calibration plots for the $v$ winds at 30 cross validation locations for 4 models: (i) Model 1 - Holland mean with no spatial covariance (ii) Model 2 - independent (iii) Model 3 - separable LMC (iv) Model 4 - non-separable LMC.

(i) No Spatial Cov

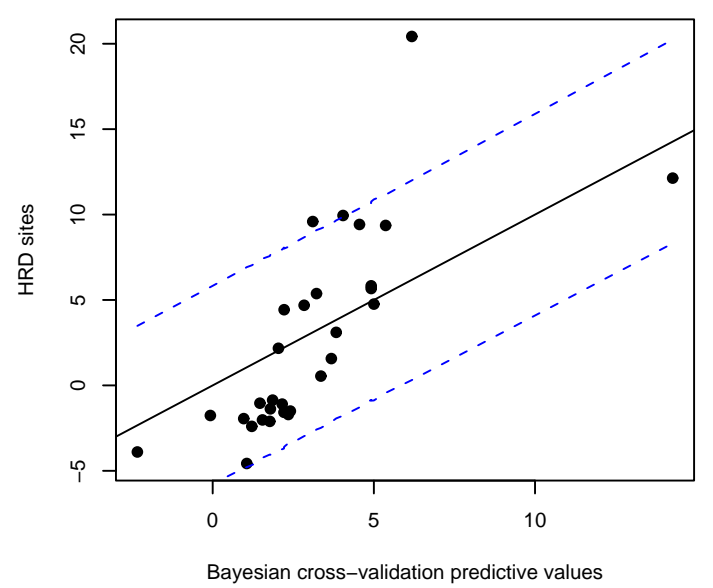

(iii) Separable

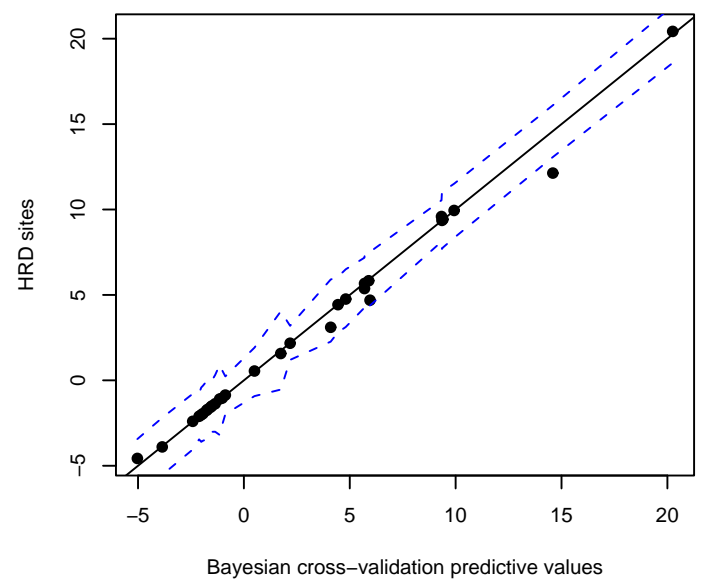

(ii) Independent

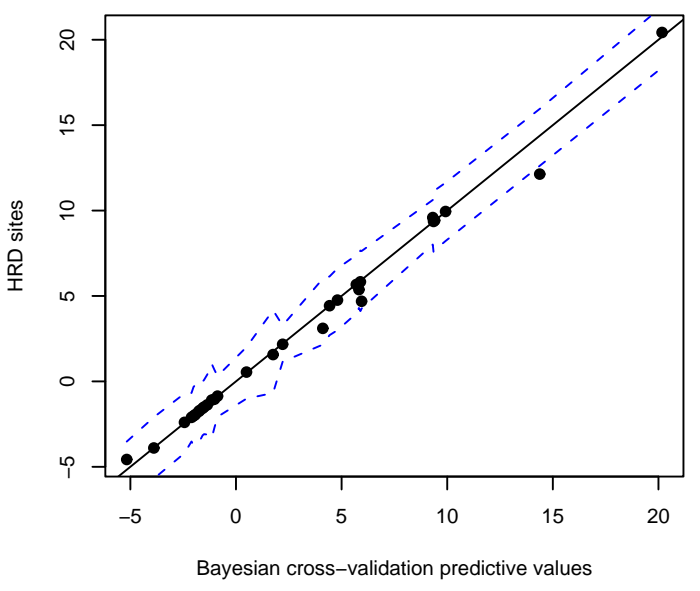

(iv) Non Separable

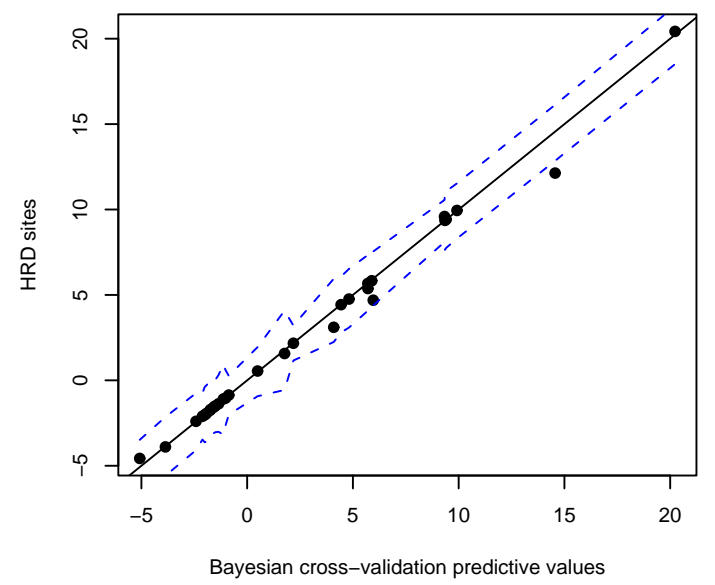


Figure 3: Map of 4 NBDC buoys within the POM domain. The remaining plots show the observed $v$ winds in $\mathrm{m} / \mathrm{s}$ at each buoy in solid black for Aug. 14th 200-1750 UTC. The dashed and dotted line is the Holland output based on fixed parameter values $(B=1.9, R \max =46)$. The dashed lines are the predicted values and the dotted lines are $95 \%$ posterior predictive intervals using Model 3.

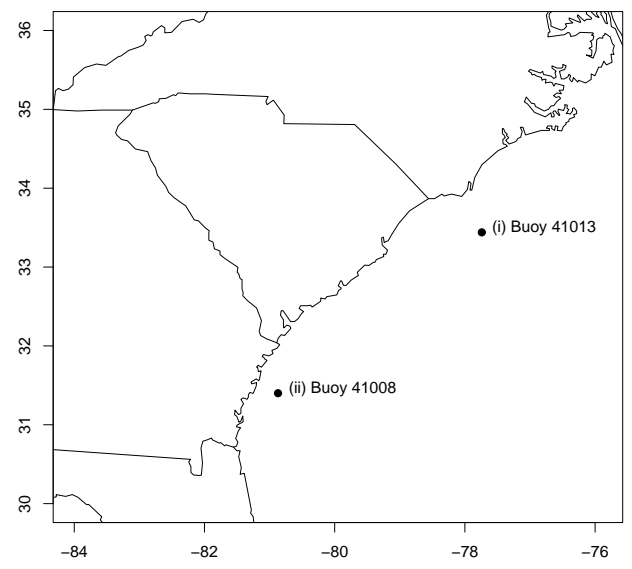

(i) Buoy 41013

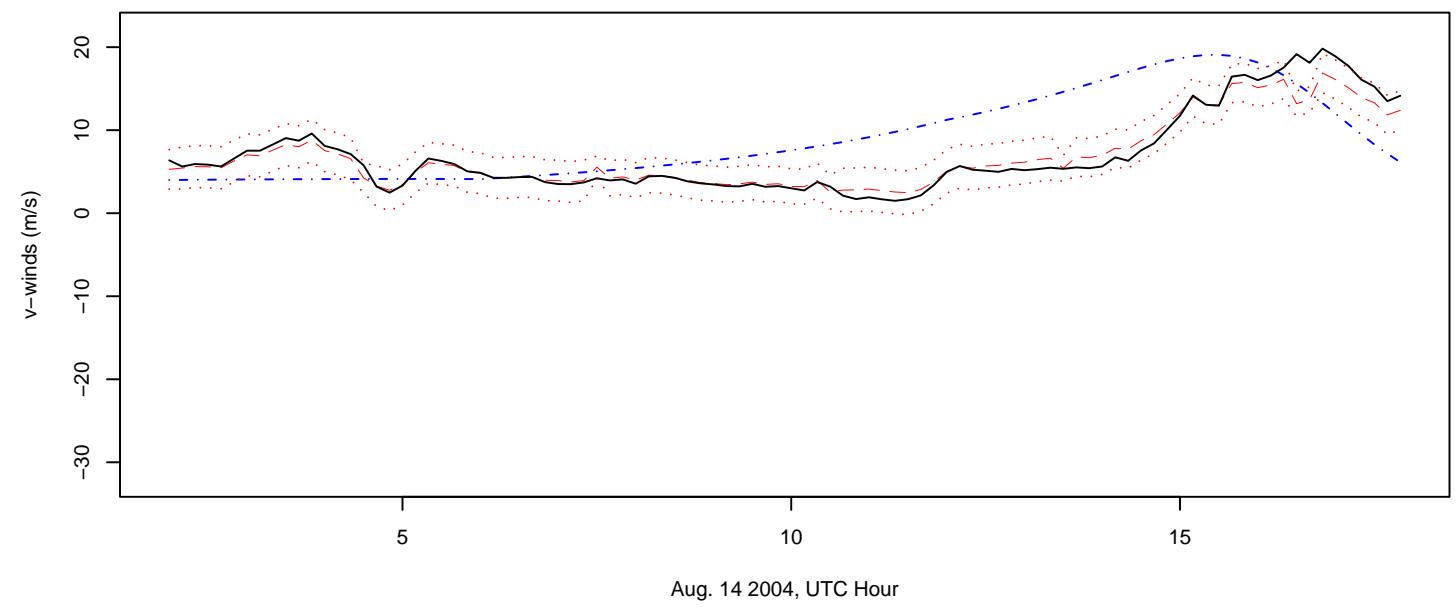

(ii) Buoy 41008

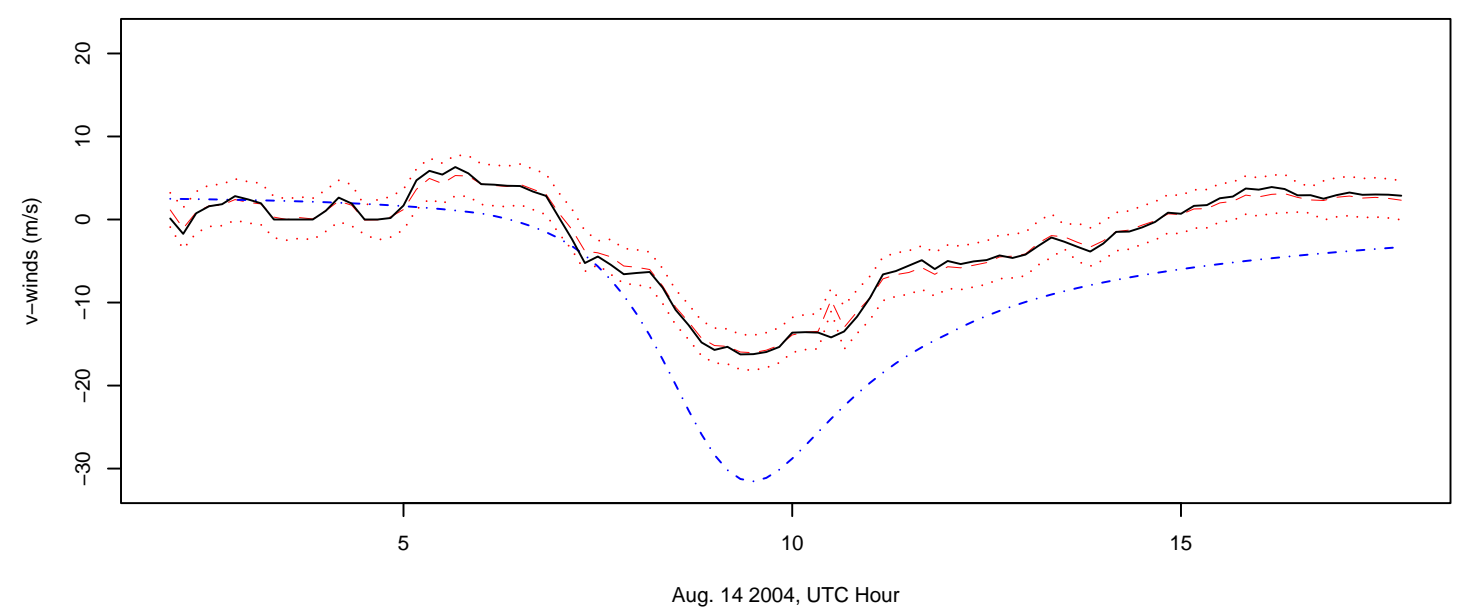


Thus it appears that in this case the empirical Bayes prediction is a reasonable approach as well as being significantly more computationally efficient.

We use the separable LMC model for prediction although, as noted earlier, the other spatial models had almost identical results in terms of prediction. At the 10 minute intervals when buoy observations are available but not the HRD datasets we use the parameter values based on the estimation results from the last available HRD time when doing the prediction. For example, at time 510 UTC the median values of the posterior predictive distribution for $\boldsymbol{\Theta}$ from time 430 UTC would be used. In this way we have estimates at 96 different time periods corresponding to 10 minute intervals between 200 to 1750 UTC on August 14th. Figure 3 shows the observed and predicted $v$ velocities for two buoys located within the ocean model domain. (Similar results were found for the $u$ component.) The dashed and dotted line is the output from the Holland model using fixed parameter values $(B=1.9, \operatorname{Rmax}=46)$. The Holland function is unrealistically smooth and tends to exaggerate the change in the winds as the hurricane approaches these locations. There is also no measure of uncertainty in the values from the Holland function. The posterior predicted values (dashed lines) match closely to the observed data at each location. The corresponding 95\%posterior predictive intervals are also included as dotted lines but we note that since an empirical Bayes approach is used here, these intervals do not account for the uncertainty in the model parameters. Still, the observed wind values fall outside of these intervals fewer than 6 of the 96 time periods in each plot.

\section{Model Diagnostics}

As noted before, we repeat the cross validation at the 30 hold out sites using the empirical Bayesian prediction. The calibration percents were all greater than 95\%. Table 3 shows the RMSPE values in meters per second for each time period. We compare the Holland model output with fixed parameters $(B=1.9, \operatorname{Rmax}=46)$, the non-spatial model and the separable LMC model using both the fully Bayesian and empirical Bayesian approaches. The Bayesian estimation of the Holland parameters decreases the RMSPE values by more than forty percent in most cases. The predictions based on the spatial model have much lower RMSPE values than the non-spatial model for all time 
periods and the values are very similar for both methods.

Table 3: RMSPE for $\mathbf{V}(\mathrm{m} / \mathrm{s})$ based on 30 cross validation sites using the Holland model output (fixed parameters), fully Bayesian prediction and the empirical Bayesian approach based on the non-spatial model (Model 1) and separable LMC model (Model 3).

\begin{tabular}{rrrrrrrr}
\hline Model & 200 UTC & 430 UTC & 730 UTC & 1030 UTC & 1330 UTC & 1630 UTC \\
\hline Holland Output & 9.57 & 7.70 & 6.33 & 7.21 & 6.53 & 6.40 \\
Full Bayes (Non-spatial) & 3.62 & 3.30 & 3.51 & 4.55 & 3.66 & 3.05 \\
Full Bayes (Spatial) & .54 & .25 & .84 & 1.79 & .64 & .18 \\
Emp. Bayes (Spatial) & .51 & .43 & 1.13 & 1.84 & .62 & .17 \\
\hline
\end{tabular}

We also repeat the prediction of the $v$ winds at the two buoys for the 96 time periods, this time without using the observed winds at these two sites. Figure 4 shows the predicted values under Model 3 (plots i-a and ii-a) and Model 1 (plots i-b and ii-b). The spatial model is still able to better capture the variability in the winds when compared to the Holland model output. The observed $v$ winds fall outside the $95 \%$ posterior prediction intervals for plots i-a and ii-a $26 \%$ and $7 \%$ of the time periods, respectively. The non-spatial model also shows an improvement over the Holland model output but the prediction intervals do not capture the observed values as often as the intervals based on the spatial model. Thus there is a clear advantage in using the statistical framework to estimate the Holland model parameters and the uncertainty in these parameters. Furthermore, there is added value in incorporating a multivariate spatial covariance through the stochastic component.

\subsection{Storm Surge Prediction}

The predicted wind fields are used as the input fields for the POM model to spin up and force the ocean model for a time between August 14th hour 0 UTC and hour 18UTC. Observed water levels were used from a series of coastal water level stations maintained by NOAA's National Ocean Service (NOS) Center for Operational Oceanographic Products and Services (CO-OPS). We have hourly data at 7 coastal sites for Hurricane Charley for the surface water elevation in meters shown in Figure 5. These observations are adjusted to account for differences in the tidal gauge locations 
Figure 4: Cross Validation Time Series Plots: Observed $v$ winds in $\mathrm{m} / \mathrm{s}$ at each buoy in solid black for Aug. 14th 200-1750 UTC. The dashed and dotted line is the Holland output based on fixed parameter values $(B=1.9, R \max =46)$. The dashed line is the predicted values and the dotted lines are 95\% credible intervals under (i-a), (ii-a) Model 3 and (i-b), (ii-b) Model 1.

(i-a) Buoy 41013: Spatial Prediction

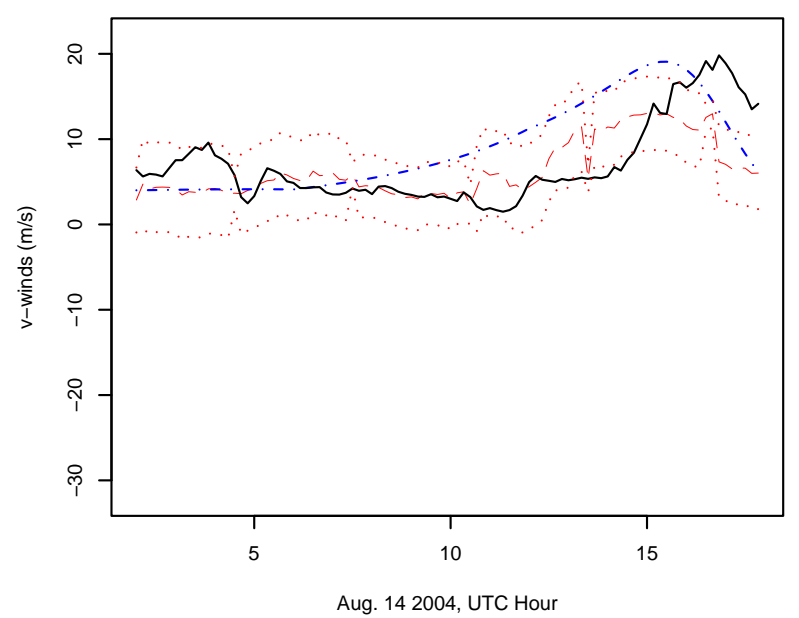

(ii-a) Buoy 41008: Spatial Prediction

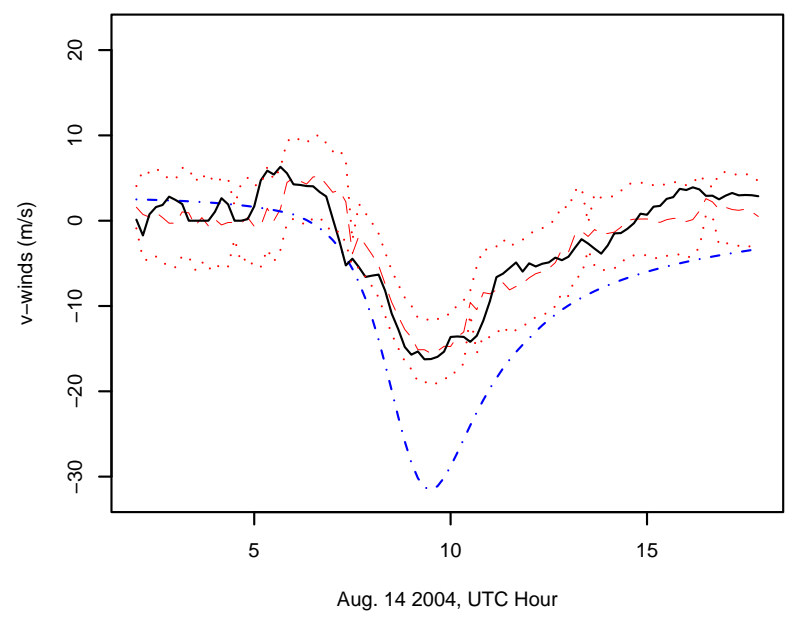

(i-b) Non-spatial Prediction

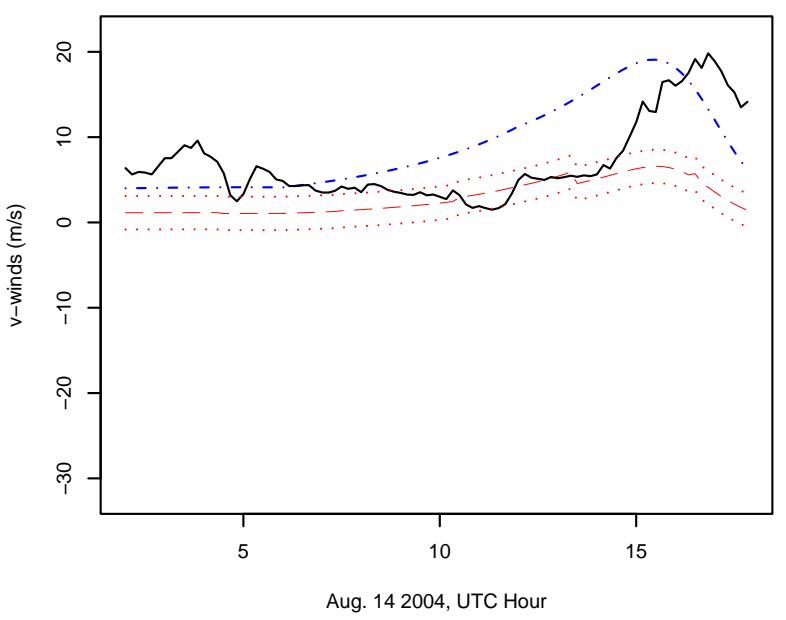

(ii-b) Non-spatial Prediction

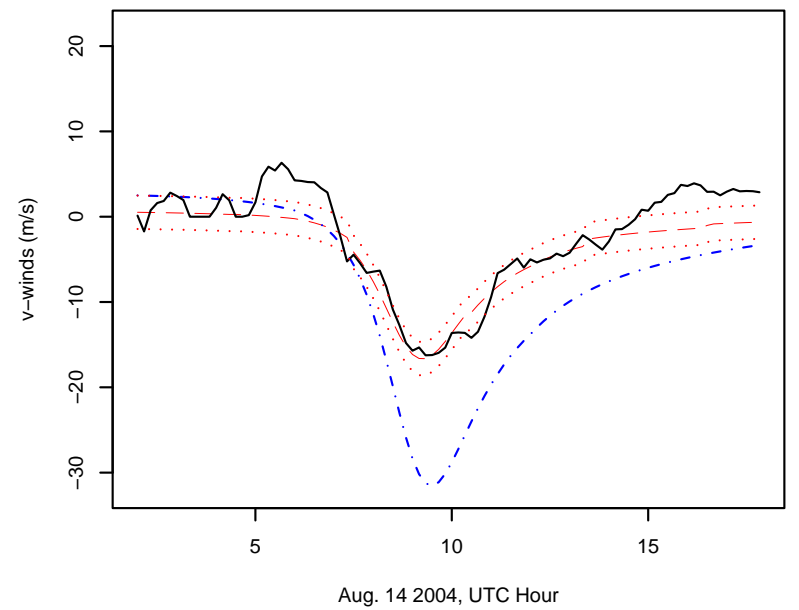


and the effect of daily tidal levels to determine the change in water elevation that can be attributed to storm surge. Figure 5 shows the observed water levels at each of these sites as Hurricane Charley moves along the coast. The Sunset Beach site shows the greatest storm surge of 1.67 meters.

The dashed and dotted line shows the predicted water elevation at each site based on hourly output of the Princeton Ocean Model under the original Holland wind forcings. Using the Holland winds with fixed parameter values the ocean model tends to greatly overestimate the storm surge at three of the four locations. The dotted line shows the predicted values using the statistically based wind estimates, $\mathbf{V}=\mathbf{V}^{H}+\tilde{\mathbf{V}}$, where $\tilde{\mathbf{V}}$ is based on Model 3 (separable LMC). The prediction error is reduced and at several of the sites these estimates tend to better capture the timing and magnitude of the peak storm surge. However the model output based on the LMC winds underestimates the greatest surge seen at the Sunset Beach site at 1600 UTC and in fact the Holland model better predicts this peak value.

\section{Discussion}

Here we present a flexible hierarchical framework to incorporate observations from different data sources with physical knowledge of hurricane surface wind fields. The multivariate spatial model proposed here allows for cross dependency between the two wind components. We found that including this dependency was important for estimation at the time periods when the hurricane structure was more asymmetric. All of the spatial models assumed that the underlying univariate processes of the stochastic residual component were Gaussian and stationary. Based on exploratory analysis we found that the Gaussian assumption was reasonable. In terms of prediction the three spatial models, Model 2, Model 3, Model 4, produced similar results and were able to produce very accurate predictions when compared to the observed data. We also saw a clear improvement in prediction when the Holland parameters were estimated using the statistical framework proposed here (Model 1) when compared to the results based on "expert knowledge".

Another approach to capture more complex spatial structures would be to allow for potential non-stationarity in the spatial processes. We expect that such a model may better fit the data 
Figure 5: Maps show the location of 7 coastal water elevation gauges and the track of Hurricane Charley on August 14th for hours 6 UTC to 18 UTC. Time series plots show observed water elevation at each site as a solid black line. The dotted line shows the predicted elevation using the predicted winds from the LMC statistical model to force the ocean model run. The dashed line shows the estimates when the ocean model is forced using the original Holland wind model.

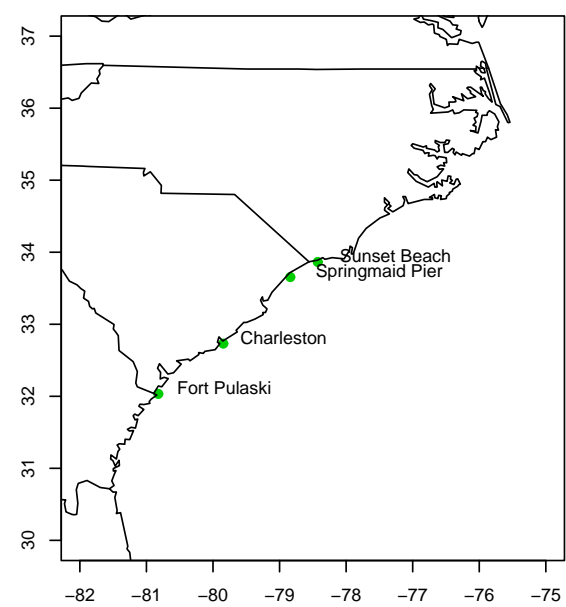

(i) Sunset Beach

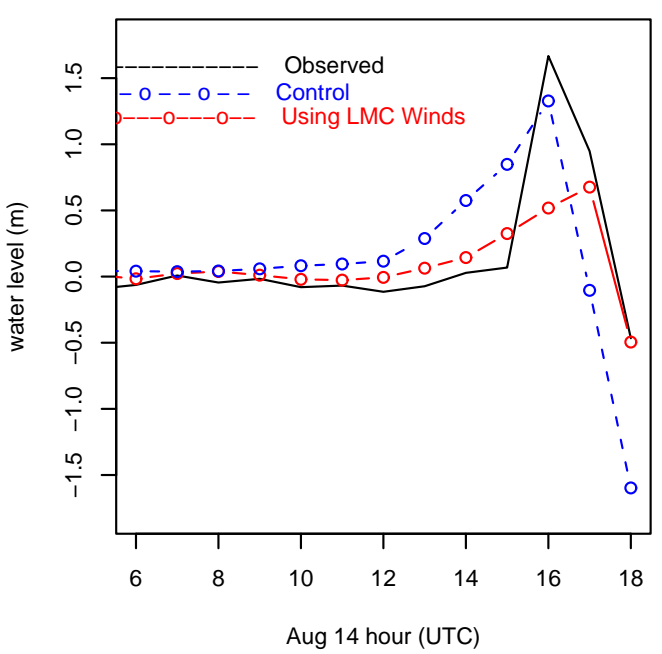

(iii) Charleston

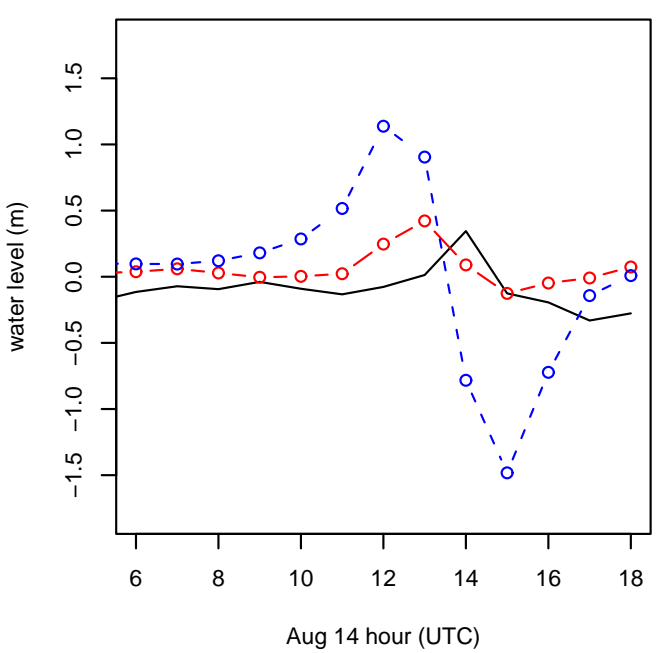

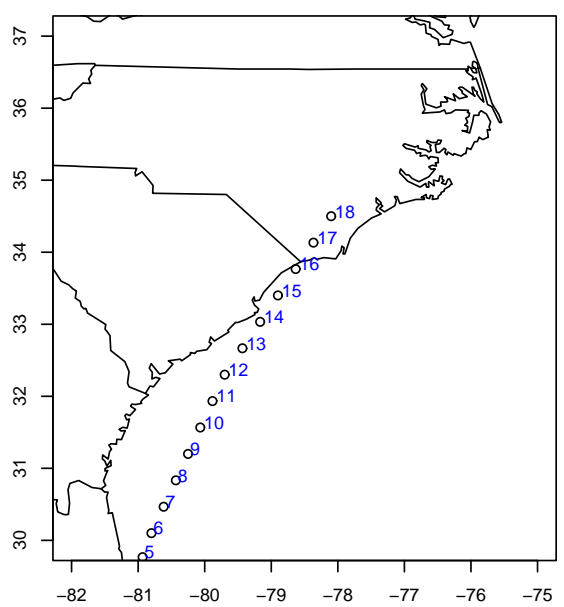

(ii) Springmaid Pier

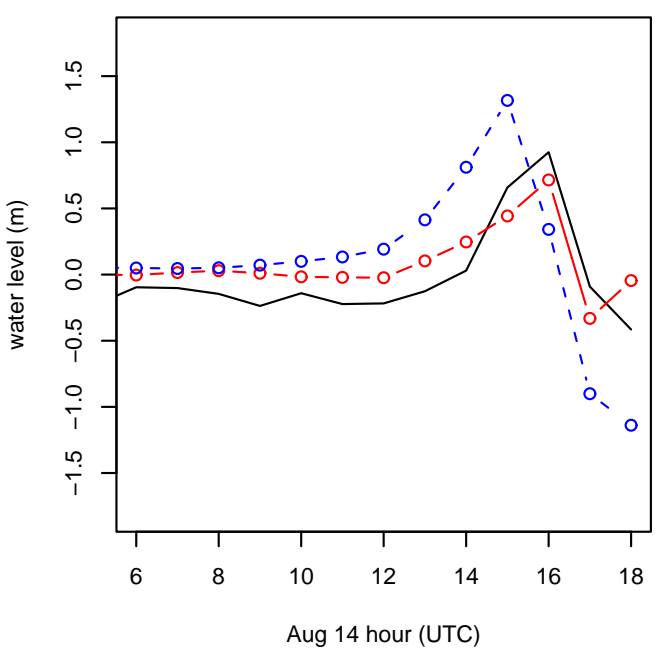

(iv) Fort Pulaski

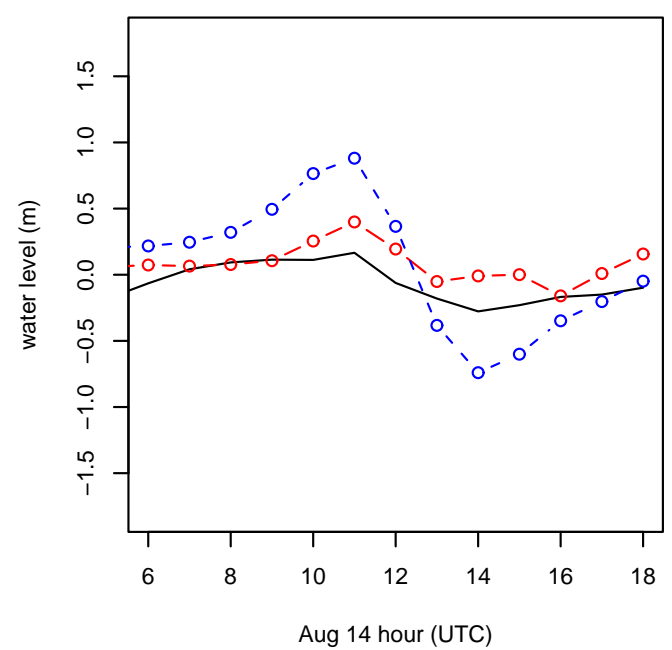


at times when the hurricane is less organized and either moving off the coast or making landfall. As noted earlier, the proposed methodology can still be used in this case by allowing for the underlying univariate processes to be non-stationary. Further analysis is necessary to test if this added complexity would be needed.

Here we used empirical Bayes prediction for computational reasons but clearly a fully Bayesian analysis provides slightly better coverage of the credible intervals. The cross validation analysis shows that prediction results are similar under either approach. Also of interest would be to consider a temporal component to allow for the evolution of the wind fields over time. For example, Wikle et al. (2001) present a hierarchical spatio-temporal model applying a first-order Markov vector autoregression model.

In applications such as storm surge prediction it is often unclear whether prediction errors are a result of simplified or neglected physical processes used in the numerical model, inaccuracies in the initial conditions or as was investigated here, errors in the forcing fields. It is likely the remaining prediction errors in the storm surge application presented here are a result of a combination of these factors. Thus changes in the ocean model itself may be necessary to reduce errors further. For example, the ocean model can be run at a higher resolution for this region and coupled with an inundation model that allows for flooding and drying in shallow coastal areas. Coastal ocean prediction for hurricane applications remains a challenging problem since we must balance potential improvement in estimation and prediction from a more sophisticated model and computational cost. In terms of computational cost, the greatest cost of the methods presented here is from the Gibbs sampling algorithm required for the estimation of the parameters of the statistical model whenever HRD data is available. This step takes approximately fifty minutes for 1000 updates using the WinBUGS software. However the prediction step using the empirical Bayes approach takes only minutes to create the gridded wind inputs based on all available buoy data at ten minute intervals. Thus after an initial lag when new HRD data becomes available it is possible to run the ocean model with updated winds for real-time storm surge prediction. 
Acknowledgements: This study is supported by the National Oceanic and Atmospheric Administration through the Carolina Coastal Ocean Monitoring and Prediction System (Caro-Coops) Program, via a subcontract from the University of South Carolina. We greatly appreciate the help and advice on the use of the ocean model application provided by members of the Coastal Fluid Dynamics Laboratory headed by Dr. Lian Xie at North Carolina State University.

\section{References}

Banerjee, S., Carlin, B. P. and Gelfand, A. E. (2004). Hierarchical modeling and analysis for spatial data. Chapman \& Hall. New York.

Berliner, L. M., Royle, J. A., Wikle, C. K. and Milliff, R. F. (1999). Bayesian methods in the atmospheric sciences. Bayesian Statistics 6 - Proceedings of the Sixth Valencia International Meeting pp. 83-100.

Blumberg, A. F. and Mellor, G. L. (1987). A description of a three-dimensional coastal ocean circulation model. in N. S. Heaps, ed., 'Three-Dimensional Coastal Ocean Models'. American Geophysical Union. Washington, D.C.. pp. 1-16.

Cripps, E., Nott, D., Dunsmuir, T. M. and Wikle, C. K. (2005). Space-time modelling of Sydney Harbour winds. Australian and New Zealand Journal of Statistics 47, 3-17.

Ezer, T. and Mellor, G. L. (1997). Data assimilation experiments in the Gulf Stream region: How useful are satellite-derived surface data for nowcasting the subsurface fields?. Journal of Atmospheric and Oceanic Technology 14, 1379-1391.

Fuentes, M. and Raftery, A. (2005). Model evaluation and spatial interpolation by Bayesian combination of observations with outputs from numerical models. Biometrics 61, 36-45.

Fuentes, M., Chen, L., Davis, J. M. and Lackmann, G. M. (2005). Modeling and predicting complex space-time structures and patterns of coastal wind fields. Environmetrics 16, 449-464.

Gelfand, A. E. and Smith, A. F. M. (1990). Sampling-based approaches to calculating marginal densities. Journal of the American Statistical Association 85, 398-409. 
Gelfand, A. E., Schmidt, A. M., Banerjee, S. and Sirmans, C. F. (2004). Nonstationary multivariate process modeling through spatially varying coregionalization (with discussion). TEST 13, 1-50.

Holland, G. (1980). An analytic model of the wind and pressure profiles in hurricanes. Monthly Weather Review 108, 1212-1218.

Houston, S. H. and Powell, M. D. (1994). Observed and modeled wind and water-level response from tropical storm Marco (1990). Weather and Forecasting 9, 427-439.

Houston, S. H., Shaffer, W. A., Powell, M. D. and Chen, J. (1999). Comparisons of HRD and SLOSH surface wind fields in hurricanes: Implications for storm surge modeling. Weather and Forecasting 14, 671-686.

Hsu, S. A. and Yan, Z. (1998). A note on the radius of maximum wind for hurricanes. Journal of Coastal Research 14, 667-668.

Large, W. G., Morzel, J. and Crawford, G. B. (1995). Accounting for surface wave distortion of the marine wind profile in low-level Ocean Storms wind measurements. Journal of Physical Oceanography 25, 2959-2971.

Peng, M., Xie, L. and Pietrafesa, J. (2004). A numerical study of storm surge and inundation in the Croatan-Albemarle-Pamlico Estuary System. Estuarine, Coastal and Shelf Science 59, 121-137.

Powell, M. D. and Houston, S. H. (1996). Hurricane Andrew's landfall in South Florida. Part II: Surface wind fields and potential real-time applications. Weather Forecast 11, 329-349.

Powell, M. D., Houston, S. H. and Reinhold, T. A. (1996). Hurricane Andrew's landfall in South Florida. Part I: Standardizing measurements for documentation of surface wind fields. Weather Forecast 11, 304-328.

Royle, J. A., Berliner, L. M., Wikle, C. K. and Milliff, R. (1999). A hierarchical spatial model for constructing wind fields from scatterometer data in the Labrador Sea. Case Studies in Bayesian Statistics Volume IV pp. 367-382.

Schmidt, A. M. and Gelfand, A. E. (2003). A Bayesian coregionalization approach for multivariate pollutant data. Journal of Geophysical Research 108, 8783. 
Spiegelhalter, D. J., Best, N. G., Calin, B. P. and van der Linde, A. (2002). Bayesian measures of model complexity and fit (with discussion). Journal of the Royal Statistical Society, Series B 64, 583-640.

Spiegelhalter, D., Thomas, A., Best, N. and Gilks, W. (1996). Bugs .5 Bayesian inference using Gibbs sampling. Manual, version ii. MRC Biostatistics Unit, Institute of Public Health. Cambridge, U.K.

Stein, M. L. (1999). Interpolation of Spatial Data. Some Theory for Kriging. Springer. New York, N.Y.

Wackernagel, H. (1998). Multivariate geostatistics: An introduction with applications. Springer. New York.

Wikle, C. K., Berliner, L. M. and Cressie, N. (1998). Hierarchical Bayesian space-time models. Environmental and Ecological Statistics 5, 117-154.

Wikle, C. K., Milliff, R. F., Nychka, D. and Berliner, L. M. (2001). Spatiotemporal hierarchical Bayesian modeling: Tropical ocean surface winds. Journal of the American Statistical Association 96, 382-397.

Xie, L., Bao, S., Pietrafesa, L., Foley, K. and Fuentes, M. (2006). A real-time hurricane surface wind forecasting model: Formulation and verification. Monthly Weather Review 134, 1355-1370.

Xie, L., Pietrafesa, L. and Peng, M. (2004). Incorporation of a mass-conserving inundatoin scheme into a three dimensional storm surge model. Journal of Coastal Research 20, 1209-1223. 